\section{BIOENGINEERING} Aids for the Disubled

from a Correspondent

THAT the successful design of an aid for the disabled depends on detailed knowledge of the behaviour of the normal was clearly illustrated at the conference on human locomotor engineering held at the University of Sussex from September 7 to 10 . The Institution of Mechanical Engineers had assembled speakers from many disciplines and discussion was both brisk and fruitful.

From a number of contributions dealing with the characterization of individual gait patterns in the various phases of the walking cycle, using electromyographic, cine-photographic or forceplate recording, it became clear that there is a need for more data about the functional anatomy of the limbs, and in particular about the exact positions of the lines of action of the various muscles relative to the centres of rotation at the relevant joints. Without this information it is difficult to calculate the force which any specific muscle exerts at a particular time or what the stresses are on the ligaments or articular facets of a joint. Quantitative descriptions of the time courses of several parameters in the gait pattern are required both for the design of the dynamics of artificial limbs and also in assessing the fitting of particular limbs to individual patients. Some of the walkway studies, such as that described by Dr M. Milner (Groote Schuur Hospital, Cape Town) and Dr T. Kasvand (National Research Council, Ottawa), now incorporate computerscanning of the cine-film to derive the paths of markers attached to the subject over the axes of rotation at the various joints.

The recordable electrical activity of the muscles proves disappointing as a guide to the magnitude of the forces, but it does give useful timing information and an ingenious technique of display has been devised by Dr D. S. Grieve and Mr P. R. Cavanagh (Royal Free Hospital, London). Instantaneous values of the angles at adjacent joints, say, knee and ankle or knee and hip, are plotted against one another during the walking cycle and the resulting locus is marked off to indicate the period during which a specific muscle is active. The patterns generated in this way can be recognized by eye and may prove useful in recording the progress of clinical conditions.

Knowledge of the normal patterns of muscle activity also provides the basis for programmed electrical stimulation of muscles to restore a lost function. Dr E. M. Sedgwick and Mr S. A. G. Chandler (University of Southampton) have developed a neat trick of feeding back the amplitude of the $M$ wave of the electromyograph in a servo to give proportional control of the number of motor units stimulated. This method overcomes the difficulty that the range of intensities that lie between threshold and maximal stimulation is small and is comparable with the variation in the threshold itself.

In the design of artificial joints for implantation, there are many conflicting requirements. Dr P. S. Walker (The Hospital for Special Surgery, New York) concluded that the chief long term problem now is the reduction of wear at the surfaces of contact. New materials need to be tried. The properties of the adhesives at present in use also leave something to be desired, particularly where tensile stresses are involved.

In the field of simulations, $\mathrm{Mr} \mathrm{D}$. C. Witt and Mr J. I. Hall (University of Oxford) have made considerable progress with their automatically stabilized powered walking device. This device is about five feet tall and stands on small feet at the ends of telescopic legs a few inches apart. The leg lengths are adjusted by hydraulic actuators to give alternating support, stability being achieved by adjusting the time of dwell on the appropriate side. They use a device modelled on the semicircular canals of the inner ear as an attitude sensor to control the switch-over. In a film, the "robot" was shown walking on the spot and it was not unbalanced when a block of wood was suddenly inserted under the descending foot. Symmetrical fore-and-aft motion of the legs has now been incorporated and the device is making its first steps in forward locomotion.

Professor J. M. Nightingale and Mr R. W. Todd (University of Southampton) described a robot hand which provides complex finely-adjusted movements of the digits to perform acts initiated by electromyographic signals from the operator (patient). The control signals that can be picked up from a patient and made available to control an external device are very severely limited in their information-carrying capacity. Adjustments of the digits to take account of the shape or fragility of the object to be grasped thus need to be delegated to an automaton which can take over the "unconscious" elements in the control task.

\title{
Measuring Epstein-Barr Virus Genomes
}

Whether or not Epstein-Barr (EB) virus has any role in the aetiology of Burkitt's lymphoma or nasopharyngeal carcinoma is still a matter of controversy and there seems little prospect of the issue being decided in the next several years. On the other hand, it is now generally accepted, chiefly as a result of the hybridization experiments done by Zur Hausen and his colleagues (Nature, 227, 245; and 228, 1056; 1970), that cell lines obtained from Burkitt lymphoma biopsies may contain detectable amounts of EB virus DNA even though these cells do not produce progeny EB virus particles. That is of course a familiar situation to tumour virologists who for the past several years have argued about precisely how many polyoma virus or Simian Virus 40 genomes are associated with the genome of a transformed cell. And now the same arguments are arising among those studying EB virus, for in next Wednesday's Nature New Biology, Nonyama and Pagano suggest that the so-called Raji line of Burkitt lymphoma cells may contain about sixty genome equivalents of EB virus DNA rather than about six equivalents, which was the estimate of Zur Hausen's group.

Zur Hausen's estimate was based on measurements of the extent to which EB virus DNA hybridizes with DNA from Raji cells and Nonyama and Pagano have measured the extent to which hot RNA, transcribed in vitro off EB virus DNA by Escherichia coli
RNA polymerase, will hybridize with Raji cell DNA. The advantage of this second technique is simply that the RNA can be made some 100 -fold hotter than EB virus DNA. Using their high specific activity RNA, Nonyama and Pagano find some 700 genome equivalents of EB virus DNA in a line of Burkitt cells which produces progeny EB virus and some sixty-two genome equivalents of EB virus DNA associated with the mitotic chromosomal DNA of the non-producer Raji line. They also detect between forty-five and a hundred genome equivalents of this viral DNA in human leukaemic and normal leukocytes culture, cells which do not carry detectable amounts of EB virus antigens. By contrast the human HeLa cell does not contain detectable amounts of EB virus DNA, and Nonyama and Pagano believe that their data support the idea that infection by EB virus is required for the continuous growth in culture of human leukocytes.

What Sugawara, Mizuno and Osato have to say in next Wednesday's Nature New Biology should also interest anybody working with EB virus. By centrifugation through discontinuous gum acacia density gradients they have separated, by virtue of differences in buoyant density, EB virus carrier cells from cells actively supporting the replication of this virus. As the active replication of the virus in carrier cells is initiated and progresses so the buoyant density of the cells seems to decrease, and their separation becomes possible. 